\title{
The Origin of Chaos in the Outer Solar System
}

\author{
N. Murray ${ }^{1}$ and M. Holman ${ }^{2}$ \\ ${ }^{1}$ Canadian Institute for Theoretical Astrophysics, \\ 60 St. George st., University of Toronto, Toronto, Ontario M5S 3H8, Canada \\ ${ }^{2}$ Harvard-Smithsonian Center for Astrophysics, \\ 60 Garden st., Cambridge, MA 02138, U.S.A.
}


Classical analytic theories of the solar system indicate that it is stable, but numerical integrations suggest that it is chaotic. This disagreement is resolved by a new analytic theory. The theory shows that the chaos among the Jovian planets results from the overlap of the components of a mean motion resonance among Jupiter, Saturn, and Uranus, and provides rough estimates of the Lyapunov time (10 million years) and the dynamical lifetime of Uranus ( $10^{18}$ years). The Jovian planets must have entered the resonance after all the gas and most of the planetesimals in the protoplanetary disk were removed. 
The predictability of planetary motions was largely responsible for the acceptance of Newton's theory of gravitation. In spite of this, Newton doubted the long term stability of the solar system. Laplace noted that the ratios $\mu$ of planetary masses $M$ to solar mass $M_{\odot}$ are small ( $\mu \sim 10^{-3}$ to $10^{-9}$ ), as are the planet's orbital eccentricities $e \sim 10^{-2}$ and inclinations $i \sim 10^{-2}$ (in radians). Neglecting terms proportional to second or higher powers of these quantities, Laplace showed that the motions of the planets were stable [1]. In this century, Arnold showed that for $\mu, e$, and $i$ of order $10^{-43}$, most planetary systems, in the sense of measure theory, are stable and undergo quasiperiodic but bounded variations in semimajor axis $(a), e$, and $i$ for each planet $(2)$. However, the values of the small quantities in our solar system are much larger than $10^{-43}$, so the applicability of Arnold's theory is uncertain. Studies over the last decade have been dominated by brute force numerical integration. Sussman and Wisdom [3], and Laskar [4], performed numerical integrations of the planet's orbits and found positive Lyapunov exponents, indicating that they are chaotic. Sussman and Wisdom also showed that integrations of the Jovian planets Jupiter, Saturn, Uranus, and Neptune are by themselves chaotic. In neither case are the variations in $a, e$ and $i$ quasiperiodic, nor is it clear that they are bounded. Are the numerical results incorrect, or are the classical calculations simply inapplicable?

We show analytically that the results of Laplace and Arnold do not apply to our solar system. The chaos seen in integrations of the outer planets arises from the overlap of the components of a three body mean motion resonance among Jupiter, Saturn, and Uranus, with a minor role played by a similar resonance among Saturn, Uranus, and Neptune. We test the theory using a suite of numerical integrations. The widths $\Delta a / a$ of the individual resonances are of order $3 \times 10^{-6}$, so that small changes in the initial conditions of the planets can lead to regular motion. This explains the puzzling dependence of Lyapunov time with integration step size seen in the outer planet integrations of Sussman and Wisdom [3]. However, the uncertainties in the initial conditions, and those introduced by our numerical model, are 
comfortably smaller than the width of the individual resonances, so our solar system is almost certainly chaotic. The resonance is extremely weak and hence easily disrupted. Torques exerted on the planets by the protoplanetary gas disk and by planetesimals were orders of magnitude larger than the resonant torques, so most of the planetesimals and all the gas must have been cleared from the outer solar system before the planets entered the resonance.

Analytic Theory. Orbital dynamics. Planets in the solar system follow nearly Keplerian orbits*. The orbit of each planet can be thought of as consisting of three nonlinear oscillators, corresponding to the three spatial directions. The Kepler problem is unusual in that all three oscillations have the same frequency. The orbital elements were chosen to take advantage of this degeneracy. The angle $l$ varies on the orbital time scale, while the angle $\varpi$ describing radial motion and the angle $\Omega$ describing vertical motion are fixed. In the actual solar system $\varpi$ and $\Omega$ are time dependent, with frequencies denoted by $g_{j}$ and $s_{j}$ respectively. These frequencies are proportional to the mass ratios $\mu$, and are consequently much smaller than the mean motion $n=d l / d t$, the time rate of change of the mean anomaly. While our model contains only the Jovian planets, we label $g_{j}$ and $s_{j}$ with $j=5,6,7$, and 8 , corresponding to the radial order of the planets in the solar system. The mean motions $n$ in units of cycles per day and the modal frequencies of the Jovian planets were determined

\footnotetext{
${ }^{0}$ The orbits have sizes and shapes described by semimajor axis $a$ and eccentricity $e$. The orientation of an orbit is described by the inclination $i$, the longitude of the ascending node $\Omega$, and the longitude of perihelion $\varpi$, while the location of the planet in the orbit is described by the mean anomaly $l$ or equivalently the mean longitude $\lambda \equiv l+\varpi$. Collectively these variables are called orbital elements. In the Kepler problem, where a single planet orbits a spherical star, all the elements of the planet except the mean longitude are fixed, which is why the elements are useful quantities. We use the masses and $a, e$, and $i$ from the JPL ephemeris DE200 (Table 1)
} 
by numerical integration of the equations of motion (Table 2). Each planet's elements vary with all the frequencies $s$ and $g$. For example

$$
e_{J} \sin \varpi_{J} \approx e_{55} \sin \left(g_{5} t+\xi_{5}\right)+e_{56} \sin \left(g_{6} t+\xi_{6}\right)+\ldots
$$

where $e_{55} \approx 0.044, e_{56} \approx 0.016$, and $\xi_{5}$ and $\xi_{6}$ are constants.

Resonances and chaos. A resonance occurs when two or more oscillators are coupled in such a way that a linear combination of their angles $\sigma \equiv \sum_{i} p_{i} \theta_{i}$ undergoes a bounded oscillation, in which case $\sigma$ is said to librate. In the sum defining $\sigma$, $i$ denotes the $i$ th oscillator and the $p_{i}$ s are (possibly negative) integers. When the oscillators are not resonant, all possible combinations of $\theta_{i}$ 's increase or decrease indefinitely, in which case $\sigma$ is said to rotate. The physical significance of a resonance is that energy is exchanged between the oscillators over a libration period, which is large compared to the oscillation period of any of the oscillators. This prolonged exchange can lead to large changes in the motion of the system. The orbit that divides regions of phase space where $\sigma$ librates from those where $\sigma$ rotates is called the separatrix.

The other bit of dynamics needed to understand our result is the notion of resonance overlap. Chaos in Hamiltonian systems, of which the motions of the planets are an example, arises when the separatrix of one resonance is perturbed by another resonance. The extent of the chaos depends on the stochasticity parameter $K$, which is a function of the of separatrix width divided by the distance between resonances. If $K$ is small, there is little chaos, but for $K>1$ the region in the immediate vicinity of the resonances is primarily chaotic (5). An orbit which, at different times, both librates and rotates must cross the separatrix, and is therefore chaotic. Another signature of chaos is that two initially nearby chaotic orbits diverge exponentially with time; in our numerical work we use both diagnostics.

Two body mean motion resonances. Two planets are said to be in a mean motion resonance when $p_{1} d \lambda_{1} / d t \approx p_{2} d \lambda_{2} / d t$. In that case conjunctions between the planets occur 
at nearly fixed locations in space. The designation "mean motion" is a little misleading, because if $p_{1} \neq p_{2}$ there is no coupling between the $(\lambda, a)$ motion of two planets that does not involve a third degree of freedom, either the radial $(\varpi, e)$ or vertical $(\Omega, i)$ motion of at least one of the planets $(6)$.

There are no two body mean motion resonances among the planets. However, there is a near mean motion resonance between Jupiter and Saturn; Jupiter makes five circuits around the sun in about the same time that Saturn orbits twice. Saturn affects the orbit of Jupiter through its gravity, described by the potential

$$
\phi=-\frac{G M_{S}}{\left|\mathbf{r}_{J}-\mathbf{r}_{S}\right|}
$$

where $M_{S}$ is the mass of Saturn, $\mathbf{r}_{J}$ and $\mathbf{r}_{S}$ are the position vectors of Jupiter and Saturn, and $G$ is the gravitational constant. To see the resonance mathematically, we expand $\mathbf{r}_{J}$ and $\mathbf{r}_{S}$ in terms of the orbital elements of the two planets, keeping only the lowest order terms:

$$
\phi=-\frac{G M_{S}}{a_{S}} \sum_{k, q, p, r} \phi_{k, q, p, r}^{(2,5)}\left(a_{S} / a_{J}\right) e_{S}^{k} e_{J}^{q} i_{S}^{p} i_{J}^{r} \cos \left[2 \lambda_{J}-5 \lambda_{S}+k \varpi_{S}+q \varpi_{J}+p \Omega_{S}+r \Omega_{J}\right] .
$$

The amplitudes $\phi_{k, p, q, r}$ can be found in classic references (7). This result shows explicitly that the gravitational coupling between two bodies on Keplerian orbits always involves either $(\varpi, e)$ or $(\Omega, i)$, so that at least three oscillators are affected. Symmetry considerations show that the integers in the argument of the cosine must sum to zero, $2-5+k+q+p+r=0$, and that $p+r$ must be even. To lowest order in the eccentricities and inclinations, the integers $k, q, p$, and $r$ are non-negative and must sum to three. The strength of the coupling is proportional to $e^{3}$ or $e i^{2}$, so this resonance is said to be of third order. Hence there are ten frequencies associated with the resonance, four involving only perihelion precession rates, such as

$$
2 \dot{\lambda}_{J}-5 \dot{\lambda}_{S}+2 \dot{\varpi}_{J}+\dot{\varpi}_{S}
$$

and six involving the precession rates of the nodal lines, including

$$
2 \dot{\lambda}_{J}-5 \dot{\lambda}_{S}+\dot{\varpi}_{J}+\dot{\Omega}_{J}+\dot{\Omega}_{S}
$$


The dot over the angles in these expressions denotes a time derivative. Each of the ten members of Eqn. (3) is referred to as a resonant term or, sometimes, as a resonance. The reason for this misuse of terminology is that, while none of the frequencies associated with these terms in our solar system vanish, they are much smaller than the mean motions of Jupiter and Saturn. As a result, the resonant terms have a strong effect on the orbits of the two planets.

Eighteenth century astronomers, unaware of the significance of these long period terms, noted a discrepancy between the predicted and observed longitude of Jupiter and Saturn. This discrepancy, known as the great inequality [8], was finally explained by Laplace. He noted that the resonant terms given by Eqn. (3) force a periodic displacement of 21 minutes of arc in Jupiter's longitude and 49 minutes of arc in Saturn's, showing that the predictions of the law of gravitation agreed with observations of the two planets.

The largest effect of Saturn's gravity on $e_{J}$ is the secular variations described by Eqn. (1). However, the most relevant component of Saturn's gravity for chaotic motion is described by Eqn. (3). This component forces much smaller variations in $e_{J} \sin \varpi_{J}$ given by

$$
\begin{aligned}
e_{J}^{2,5} \sin \varpi_{J} \approx & \frac{\mu_{S}}{\left(2-5 n_{S} / n_{J}\right)} \frac{a_{J}}{a_{S}} \sum_{p>0} \phi_{k, p, q, r}^{(2,5)} e_{S}^{k} e_{J}^{p-1} i_{J}^{q} i_{S}^{r} \\
& \times \sin \left[2 \lambda_{J}-5 \lambda_{S}+k \varpi_{S}+(p-1) \varpi_{J}+q \Omega_{J}+r \Omega_{S}\right] .
\end{aligned}
$$

The largest variation in $e_{J}^{2,5}$, corresponding to $k=2, p-1=q=r=0$ and $\phi_{2,1,0,0} \approx 9.6$, has an amplitude of about $3.5 \times 10^{-4}$. Our numerical integrations yield $3.7 \times 10^{-4}$, consistent within the errors introduced by keeping only the highest order term in $e$. This variation in $e_{J}$ plays a central role in producing chaos among the outer planets.

There are other two body near mean motion resonances in the solar system. Of particular relevance here is the $7 \lambda_{U}-\lambda_{J}$ near resonance between Jupiter and Uranus. The potential 
experienced by Uranus is

$$
\phi=-\frac{G M_{J}}{a_{U}} \sum_{k, q, p, r} \phi_{k, q, p, r}^{(7,1)} e_{J}^{k} e_{U}^{q} i_{J}^{p} i_{U}^{r} \cos \left[\lambda_{J}-7 \lambda_{U}+k \varpi_{J}+q \varpi_{U}+p \Omega_{J}+r \Omega_{U}\right] .
$$

To lowest order (sixth) in $e$ and $i$, there are 44 terms. The coefficients $\phi_{k, q, p, r}^{(7,1)}$ range from $\sim 10^{-3}$ to $\sim 10$. By itself this resonance has little effect on the dynamics of the solar system.

Three body mean motion resonances. Now consider the fact that $e_{J} \sin \varpi_{J}$ varies; substituting (6) into (7), we find the potential experienced by Uranus due to the non-Keplerian orbit of Jupiter;

$$
\begin{aligned}
\phi \approx & -\frac{G M_{J}}{a_{U}} \mu_{S} \epsilon_{J S}^{-1} \alpha_{J S} \sum_{p=0}^{5}(6-p) \phi_{6-p, p, 0,0}^{(7,1)} \phi_{2,1,0,0}^{(5,2)} e_{J}^{5-p} e_{U}^{p} e_{S}^{2} \\
& \times \sin \left[3 \lambda_{J}-5 \lambda_{S}-7 \lambda_{U}+7 \varpi_{J}+p\left(\varpi_{U}-\varpi_{J}\right)+2 \varpi_{S}\right],
\end{aligned}
$$

where $\alpha_{J S}=a_{J} / a_{S} \approx 0.55$ and $\epsilon_{J S}=\left|2-5\left(n_{S} / n_{J}\right)\right| \approx 1.3 \times 10^{-2}$. For simplicity we have ignored terms involving the inclinations and kept only terms proportional to $e_{S}^{2}$. This three body mean motion resonance is second order in the masses of the planets (both $\mu_{J}$ and $\mu_{S}$ appear) and seventh order in $e$.

Using the frequencies in Table 1, and accounting for terms involving $i$, we find a mixed $e-i$ resonance at $a_{U} \approx 19.21796 \mathrm{AU}$ associated with the argument

$$
3 \lambda_{J}-5 \lambda_{S}-7 \lambda_{U}+7 g_{6} t+2 s_{7} t
$$

We find a cluster of eccentricity resonances centered at $a_{U} \approx 19.2163 \mathrm{AU}$ with argument

$$
3 \lambda_{J}-5 \lambda_{S}-7 \lambda_{U}+(2-q) g_{5} t+7 g_{6} t+q g_{7} t
$$

where $0 \leq q \leq 2$, and at $a_{U} \approx 19.2193 \mathrm{AU}$ with argument

$$
3 \lambda_{J}-5 \lambda_{S}-7 \lambda_{U}+(3-q) g_{5} t+6 g_{6} t+q g_{7} t
$$

where $0 \leq q \leq 3$. At the present epoch the JPL ephemeris DE200 has $a_{U} \approx 19.21895 \mathrm{AU}$ 
For simplicity we have described only one type of term in the potential experienced by Uranus, that due to the influence of Jupiter as it moves in the potential of the Sun and Saturn, as reflected in $e_{J} \sin \varpi_{J}$. There are similar contributions to the potential due to variations in Jupiter's other orbital elements. Furthermore, there are weaker resonances due to the gravity of Saturn, moving on an orbit perturbed by Jupiter, and acting on Uranus. Finally there are much smaller terms due to the direct perturbations of Uranus by Saturn and Jupiter moving on their unperturbed Keplerian orbits.

We also find three body resonances with arguments containing

$$
3 \lambda_{S}-5 \lambda_{U}-7 \lambda_{N}+7 g_{6} t+(2-q) s_{7} t+q s_{8} t
$$

at 19.2187 $\mathrm{AU} \lesssim a_{U} \lesssim 19.2195 \mathrm{AU}$. The strength of the resonance is smaller than that of the resonance involving Jupiter by the ratio $\left(\mu_{N} / \mu_{J}\right)\left(\epsilon_{J S} / \epsilon_{S U}\right) \approx 3 \times 10^{-3}$.

Overlapping resonances. The overlap of the individual resonances produces chaos among the outer planets. The width of a typical component resonance is

$$
\frac{\Delta a}{a_{U}}=8 \sqrt{(6-p) \phi_{6-p, p, 0,0}^{(7,1)} \phi_{2,1,0,0}^{(2,5)} \frac{\alpha}{3 \epsilon_{J S}} \mu_{J} \mu_{S} e_{J}^{5-p} e_{U}^{p} e_{S}^{2}} \approx 2 \times 10^{-6},
$$

or $\Delta a \approx 8 \times 10^{-5} \mathrm{AU}$. We must substitute powers of either $e_{55}$ or $e_{56}$ for $e_{J}^{5-p}$, depending on the resonant argument. This resonance width is comparable to the radius of Uranus. The libration period is

$$
T_{0}=T_{U} / \sqrt{147(6-p) \phi_{6-p, p, 0,0}^{(7,1)} \phi_{2,1,0,0}^{(2,5)} \frac{\alpha}{\epsilon_{J S}} \mu_{J} \mu_{S} e_{J}^{5-p} e_{U}^{p} e_{s}^{2}} \approx 10^{7} \text { years. }
$$

The precession frequencies $g_{5}$ and $g_{7}$ determine the distance between the component resonances; we find

$$
\frac{\delta a}{a_{U}} \approx \frac{4 \pi}{21}\left(\frac{g_{5}-g_{7}}{n_{U}}\right) \approx 7 \times 10^{-6}
$$

The stochasticity parameter is

$$
K \equiv\left(\pi \frac{\Delta a}{\delta a}\right)^{2}
$$


Using Eqns. (13) and (15) in Eqn. (16), we see that $K \gtrsim 1$, so the motion is marginally chaotic. Then the Lyapunov time (the inverse of the Lyapunov exponent) is given by $T_{L} \lesssim T_{0}$ (9).

The chaotic nature of the system ensures that the angles in the perturbing potential (8) experienced by Uranus are essentially random variables. These chaotic perturbations force Uranus's $e$ to undergo a random walk, exploring all values between 0 and $e_{\text {cross }} \approx 0.5$; for $e>e_{\text {cross }}$ Uranus will suffer close encounters with Saturn, and may be ejected from the solar system. The time for this to occur is of order (9)

$$
T_{\text {cross }} \approx 6 \times 10^{17}\left(\frac{0.05}{e_{\text {cross }}}\right)^{p} \text { years }
$$

where $p$ is the exponent of $e_{U}$ in Eqn. (8). This estimate is uncertain by a large factor, possibly by one or two orders of magnitude, but it is clear that Uranus will be with us for a long time. The resonance closest to the actual value of $a_{U}$ has $p=0$.

The discovery that the great inequality was due to the $2: 5$ near resonance between Jupiter and Saturn clearly had a strong affect on Laplace's views regarding determinism. We find it ironic that the $2: 5$ resonance plays such a strong role in producing chaos among the outer planets, thereby placing a limit on our ability to state the positions of the Jovian planets in the distant future. The fact that Laplace was the first astronomer to identify a three body resonance in the solar system, involving three of the Galilean satellites, only heightens the irony. More recently, three body resonances were shown to be responsible for much of the chaos seen in integrations of asteroids (10).

Numerical integrations. In order to test our theory, we have integrated the equations of motion for the four Jovian planets using a symplectic integrator (12). We chose this simplified model rather than including all nine planets in order to isolate the effects of the giant planets. To account in a crude way for the effects of the terrestrial planets, we enhanced the mass of the sun by the their mass, roughly a part in $6 \times 10^{-6}$. This ensures that the 
location of resonances between the Jovian planets is shifted by an amount which is second order in this mass ratio, roughly $3 \times 10^{-11}$. This is much smaller than the uncertainty in the orbital elements of the planets. The orbital elements, which provide the initial conditions for our integrations, are known to a relative accuracy of a few parts in 10 million. For example, $\Delta a / a \sim 2 \times 10^{-7}$ (600km for Uranus) (11), much smaller than the size of the resonances.

To determine whether the evolution was chaotic, we measured the Lyapunov time by comparing pairs of integrations in which the initial conditions differed by 1.5 millimeters in the $x$ coordinate of Uranus. Using the DE200 ephemeris from JPL, we confirm the result of Sussman and Wisdom (3) that the four Jovian planets are chaotic. We find a Lyapunov time of about 7 million years, consistent with our analytic result and with Sussman and Wisdom's result of about 5 million years, given that it is difficult to measure Lyapunov times with an accuracy much better than a factor of 2 .

To check the robustness of this conclusion, we have carried out integrations in which we varied the initial $a_{U}$ in ten steps of $300 \mathrm{~km}$; the largest displacement was $\pm 1,500 \mathrm{~km}$, about twice the uncertainty in the JPL ephemeris. We employed symplectic correctors (12) to ensure that the relative energy errors were less than $10^{-9}$, much smaller than the uncertainties in the initial conditions. In all these integrations we found that the orbits were chaotic.

To test the prediction that the motion is marginally chaotic, we carried out various surveys of the dynamics of the Jovian planets in which all the initial orbital elements except $a_{U}$ were held fixed (14). The integration time in each survey was 200 million years. In our first survey we varied the initial value of $a_{U}$ in steps of $0.01 \mathrm{AU}$ between 18.9789 and 19.3990AU. We found that between 19.18 and 19.399 AU more than $80 \%$ of the orbits are regular. Subsequently we conducted a survey in which $a_{U}$ was varied in steps of $0.0001 \mathrm{AU}$ between 19.2141 and 19.2209. The resulting Lyapunov times are plotted as a function of 
the initial semimajor axis $a_{U}$ in Figure 1 . We plot a point at $10^{8}$ years, corresponding to the integration time, if the orbit appeared to be regular. The location of our solar system as represented in the DE200 ephemeris is indicated by the vertical line in the figure. From 18.9789 to 19.15 AU we find a strongly chaotic region, with Lyapunov times ranging from 25,000 to 2 million years. Examination of the resonant argument $\lambda_{U}-2 \lambda_{N}+\varpi_{N}$ reveals that from 18.9789 to about $19.13 \mathrm{AU}$ our pseudo-Uranus is in a $1: 2$ mean motion resonance with Neptune. From 19.13 to 19.17 AU pseudo-Uranus is in the 7 : 1 mean motion resonance with Jupiter described by Eqn. (17), with a Lyapunov time ranging upwards from 100,000 years. There are four other chaotic regions visible in Figure 1, centered at $a_{U}$ of 19.219, 19.26, 19.29, and 19.34 AU. All of these regions are associated with three-body resonances.

The dynamics in the region from 19.21 to $19.225 \mathrm{AU}$ (Figure 2) is controlled by the $3 \lambda_{J}-5 \lambda_{S}-7 \lambda_{U}$ three-body resonance described in Eqn. (8). We can see the effects of the individual resonant terms. For $a_{U}<19.218$ AU the resonances are isolated by regular regions, indicating that the resonance widths are slightly smaller than the distance between resonances. For $a_{U} \gtrsim 19.218$ AU nearly all the orbits have finite Lyapunov times, indicating that the individual resonances overlap completely. Figure 3 shows the resonant angle $3 \lambda_{J}-$ $5 \lambda_{S}-7 \lambda_{U}+3 g_{5} t+6 g_{6} t$ (the $q=0$ case of eq. (11)) for $a_{U}=19.21908$, about one planetary radius larger than the value of $a_{U}$ used in the DE200 ephemeris. It alternates between libration, with a period of about 20 million years, and rotation, indicating that the orbit is crossing the separatrix of the resonance and confirming the chaotic nature of the orbit. In addition to the $3 \lambda_{J}-5 \lambda_{S}-7 \lambda_{U}$ resonance, there is a resonant term involving Saturn, Uranus, and Neptune. Our calculations suggest that this resonance is responsible for the chaotic zones at 19.29 and 19.34, and plays a strong role in the chaotic zone at 19.26.

Integrations of simpler models. In another survey, we set $i=0$ for all four Jovian planets and again varied $a_{U}$ in steps of 0.01 between 18.9789 and $19.3990 \mathrm{AU}$, and in steps 
of 0.0001 between 19.2141 and 19.2209AU. The general appearance is similar to that of Figure 1, showing that inclination resonances are not essential to produce chaos among the Jovian planets. However, the chaotic region near $a_{U}=19.219$ AU is not quite so extensive, and the resonances appear to be isolated, like those with $a_{U}<19.218 \mathrm{AU}$ in Figure 2. In yet another survey, we removed Neptune. The chaotic region at $a_{U} \approx 19.00 \mathrm{AU}$ vanishes, but the chaos associated with $7 \lambda_{U}-\lambda_{J}$ remains. Similarly, the chaos at $a_{U}=19.29 \mathrm{AU}$ and 19.34 $\mathrm{AU}$ is no longer present. However a chaotic region at $a_{U}=19.219 \mathrm{AU}$ and a very small chaotic region at 19.25 AU remain. The feature near $a_{U}=19.219 \mathrm{AU}$ is even less extensive than in the planar case, indicating that the effects of the $3 \lambda_{S}-5 \lambda_{U}-7 \lambda_{N}$ resonance are more important than the effects of inclination resonances involving Jupiter. Finally, a survey in which Neptune is removed and the remaining Jovian planets orbit in the same plane reveals no chaotic motion outside the $7 \lambda_{U}-\lambda_{J}$ resonance. Apparently eccentricity resonances involving only the inner three Jovians do not quite overlap. They must act in concert either with inclination resonances or with three-body resonances involving Neptune to produce detectable chaotic regions.

The epoc of resonance capture. Uranus probably did not form in the current resonance. Planet formation is believed to occur in disks around young stars. Evidence for such disks, which have lifetimes around ten million years, is now abundant, including visible, infrared and millimeter observations of disks around young stars (15). The observations show that the disks contain both gas and particulate matter. The existence of our own asteroid and Kuiper belts, as well as of comets, suggest that protostellar disks contain larger bodies as well. Current understanding of the planet formation process suggests that planets migrate over substantial distances early in the history of a planetary system. Goldreich and Tremaine (16) showed that torques produced by interactions between a gas disk and a planet can cause large scale planet migrations on timescales of tens to hundreds of thousands of years. Interactions between asteroids or comets and planets can also cause planet migrations 
(17)]. The recent discovery (18) of Jupiter-mass objects in short period (4 day) orbits around nearby stars strongly suggest that planet migration is common.

We can compare the torques exerted on Uranus by the different processes. Jupiter and Saturn currently exert a resonant torque on Uranus given by

$$
T_{r e s} \approx 100\left(\frac{G M_{\odot} M_{U}}{a_{U}}\right) \mu_{J} \mu_{S}\left(\frac{e_{J}^{5-p} e_{U}^{p} e_{S}^{2}}{\epsilon_{J S}}\right)
$$

The torque exerted on proto-Uranus by the gas disk in which it formed is

$$
T_{\text {gas }} \approx 5.6\left(\frac{G M_{\odot} M_{U}}{a_{U}}\right) \mu_{U} \mu_{g} m_{\max }^{3}
$$

(16). In this expression the quantity $m_{\max }$ is a measure of the gap in the gas disk produced by Uranus. If no such gap formed, the torque produced by the gas disk is even larger. The minimum mass of the solar nebula is about 10 Jupiter masses, so $\mu_{g} \equiv M_{\text {gas disk }} / M_{\odot} \approx 0.01$. The torque produced by interactions between Uranus and a planetesimal disk is

$$
T_{\text {planetesimal }} \approx\left(\frac{G M_{\odot} M_{U}}{a_{U}}\right)\left(\frac{M_{d}}{M_{U}}\right)\left(\frac{T_{U}}{T_{\text {clear }}}\right)
$$

where $M_{d}$ is the total mass of the planetesimals that interact with Uranus, $T_{U} \approx 80$ years is the orbital period of Uranus, and $T_{\text {clear }} \approx 10^{7}$ years is the time for Uranus to clear the planetesimal disk. In units of $G M_{\odot} M_{U} / a_{U}$ the torques are $T_{r e s} \sim 10^{-11}, T_{\text {gas }} \sim 10^{-3}$, and $T_{\text {planetesimal }} \sim 10^{-6} M_{d} / M_{U}$. The planets remain in resonance only if $T_{\text {res }} \gtrsim T_{\text {gas }}$ and $T_{\text {res }} \gtrsim T_{\text {planetesimal }}$. Clearly, Uranus must have been trapped in the resonance after the gas disk dissipated. Similarly, most of the planetesimal disk must be removed before the final trapping can occur. 
Table 1. Masses, in units of the solar mass $M_{\odot}$, and the current semimajor axes $a$ (in $\mathrm{AU})$, eccentricities $e$ and inclinations $i$ of the orbits of the giant planets. Data taken from JPL ephemeris DE200.

\begin{tabular}{llccc}
\hline \hline Planet & $\mu \equiv M / M_{\odot}$ & $a(\mathrm{AU})$ & $e$ & $i$ (radians) \\
\hline Jupiter & $9.548 \times 10^{-4}$ & 5.207 & 0.04749 & 0.02277 \\
Saturn & $2.859 \times 10^{-4}$ & 9.553 & 0.05274 & 0.04338 \\
Uranus & $4.355 \times 10^{-5}$ & 19.219 & 0.04641 & 0.01348 \\
Neptune & $5.178 \times 10^{-5}$ & 30.111 & 0.00820 & 0.03089 \\
\hline
\end{tabular}


Table 2. Orbital frequencies of the giant planets, in cycles per day. Data from our numerical integrations.

\begin{tabular}{cccc}
\hline \hline Planet/mode & $n / 2 \pi\left(\right.$ days $\left.^{-1}\right)$ & $g\left(\right.$ days $\left.^{-1}\right)$ & $s\left(\right.$ days $\left.^{-1}\right)$ \\
\hline 5 & $2.308 \times 10^{-4}$ & $8.967 \times 10^{-9}$ & 0.0 \\
6 & $9.294 \times 10^{-5}$ & $5.965 \times 10^{-8}$ & $-5.564 \times 10^{-8}$ \\
7 & $3.259 \times 10^{-5}$ & $6.520 \times 10^{-9}$ & $-6.328 \times 10^{-9}$ \\
8 & $1.662 \times 10^{-5}$ & $1.420 \times 10^{-9}$ & $-1.460 \times 10^{-9}$ \\
\hline
\end{tabular}




\section{REFERENCES}

1. P. S. Laplace, Traite de Mecanique Celeste (Paris, 1799-1825).

2. V. I. Arnold, Russ. Math. Surv. 18, 85 (1961).

3. G. J. Sussman and J. Wisdom, Science 241, 433 (1988); 257, 56 (1992).

4. J. Laskar, Nature 338, 237 (1989);

5. B. V. Chirikov, Phys. Rep. 52, 263 (1979); A. J. Lichtenberg and M. A. Lieberman, Regular and Chaotic Dynamics (Springer-Verlag, New York, 1992).

6. M. Holman and N. Murray, Astron. J. 112, 1278 (1996).

7. B. Peirce, Astron. J. 1, 1 (1849); U.-J. Le Verrier, Annales de L'Observ. Imp. de Paris 1, 1 (1855); a modern computer algebraic expansion to eighth order is given by C. D. Murray and D. Harper, Expansion of the planetary disturbing function to eighth order in the individual orbital elements QMW Maths Notes, School of Mathematical Sciences, London (1993).

8. F. Moulton, An Introduction to Celestial Mechanics, (Dover, New York, 1970), p361.

9. N. Murray and M. Holman, Astron. J. 114, 1246 (1997).

10. N. Murray, M. Holman, M. Potter, Astron. J. 116, 2583 (1998); A. Morbidelli and D. Nesvorney, Astron. J. 116, 3029 (1998); for example, our integrations of asteroid 7690 Sackler show that it is in a three body resonance involving the asteroid, Jupiter, and Saturn.

11. Personal communication from Myles Standish.

12. J. Wisdom and M. Holman, Astron. J. 102, 1528 (1991). 
13. J. Wisdom, M. Holman, J. Touma "Symplectic correctors", Proceedings of the Integration Methods in Classical Mechanics Meeting, Waterloo, October 14-18, 1993., Fields Institute Communications 10, 217 (1996).

14. A similar survey is reported in G. D. Quinlan in Chaos, resonance and collective dynamical phenomena in the solar system, IAU symposium 152, Angra dos Reis, Brazil, 15-19 July 1991 (Kluwer, Dordrecht, 1992).

15. See, for example, S. V. W. Beckwith, A. Sargent, R. S. Chini, and R. Guesten, Astron. J. 99, 924 (1990); K. R. Stapelfeldt et al., Astrophys. J. Lett. 502, 65 (1998); A. Dutrey et al., Astron. Astrophys. 338 L63 (1998).

16. P. Goldreich and S. Tremaine, Astrophys. J. 241, 425 (1980).

17. J. A. Fernandez and W.-H. Ip, Icarus 58, 109 (1984); Malhotra, R., Nature 365, 819 (1993); Malhotra, R., Astron. J. 110, 420 (1995); Murray, N., Hansen, B., Holman, M., and Tremaine, S., Science 279, 69 (1998).

18. M. Mayor and D. Queloz, Nature 378, 355 (1995); D.W. Latham, R.P. Stefanik, T. Mazeh, M. Mayor, G. Burki, Nature 339, 38 (1989); G.W. Marcy and R.P. Butler, Astrophys. J. 464, L147 (1996); R.P. Butler, G.W. Marcy, E. Williams, H. Hauser, P. Shirts, Astrophys. J. 474, L115 (1997); R.W. Noyes et al., Astrophys. J. 483, L111 (1997); see also erratum in Astrophys. J., 487, L195 (1997).

19. We thank B. Gladman and J. Wisdom for helpful conversations. This research was supported by NSERC of Canada. 
Fig. 1. The Lyapunov time $T_{L}$ as a function of initial $a_{U}$. The initial orbital elements of the planets are taken from DE200, except for $a_{U}$, which is varied. There are chaotic two body resonances at $a_{U} \approx 19.00$ and $19.12 \mathrm{AU}$ involving Neptune and Jupiter, respectively. There are also chaotic regions associated with three body mean motion resonances at $a_{U} \approx 19.18$, 19.25, 19.29, and 19.34 AU. These involve either Jupiter, Saturn, and Uranus, or Saturn, Uranus and Neptune. The solid vertical line shows the actual location of Uranus.

Fig. 2. A close up of Figure 1 around the actual value of $a_{U}$. Between 19.216 and 19.218 AU we find the individual eccentricity resonances associated with the resonant argument $3 \lambda_{J}-5 \lambda_{S}-7 \lambda_{U}+q g_{5}+7 g_{6} t+(2-q) g_{7} t$, which do not quite overlap. The resonances associated with the argument $3 \lambda_{J}-5 \lambda_{S}-7 \lambda_{U}+q g_{5}+6 g_{6} t+(3-q) g_{7} t$ lie between 19.218 and 19.221 AU.

Fig. 3. The resonant argument $3 \lambda_{J}-5 \lambda_{S}-7 \lambda_{U}+3 g_{5} t+6 g_{6} t$ in the case $a_{U}=19.21908$, about one planetary radius larger than the actual value of $a_{U}$. The libration period is $T_{0} \approx 20,000,000$ years. A transition from libration to rotation occurs near 60 million years. A longer lasting transition from libration to rotation occurs at 160 million years. The Lyapunov time was measured to be about 7 million years. 


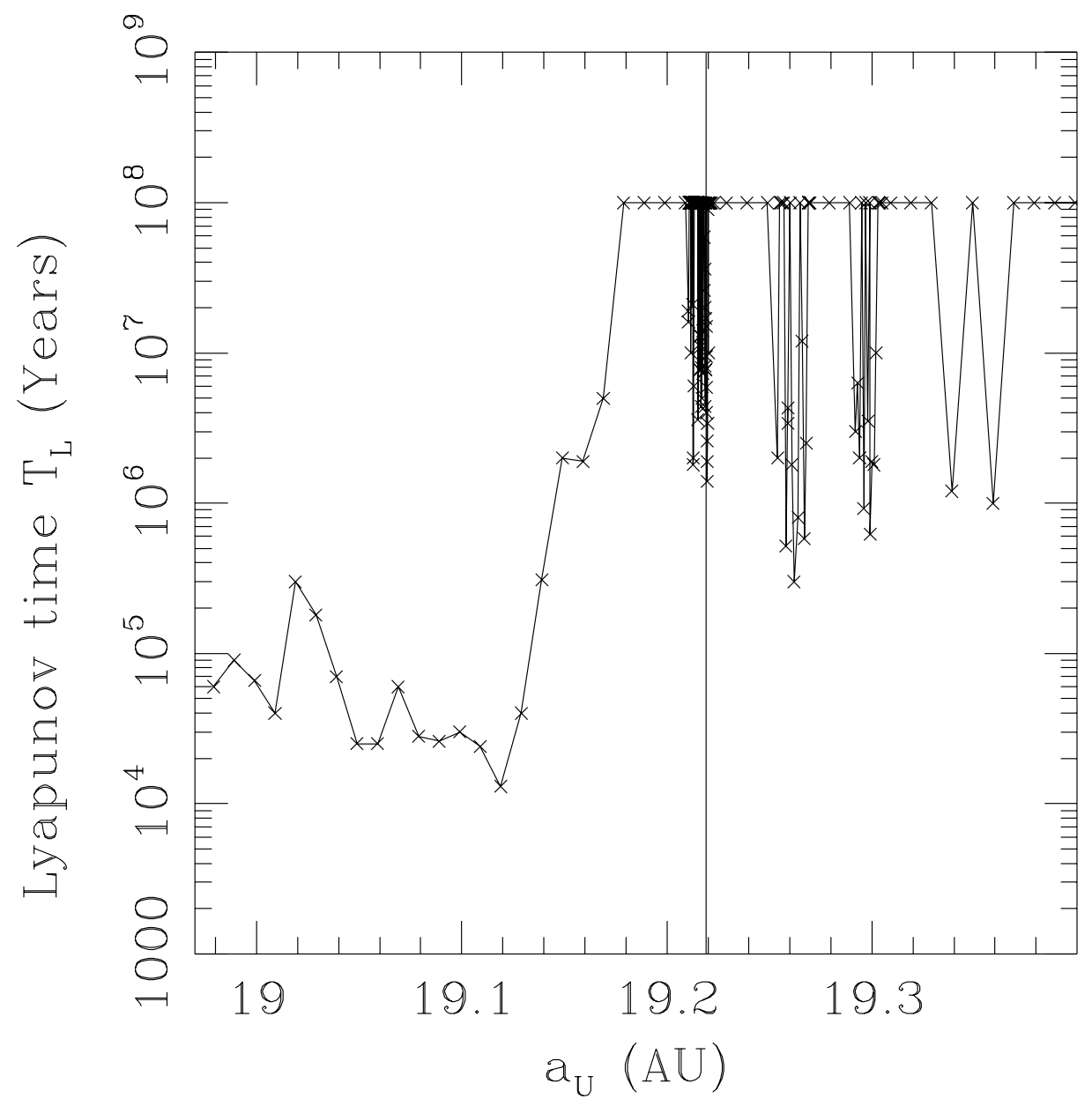




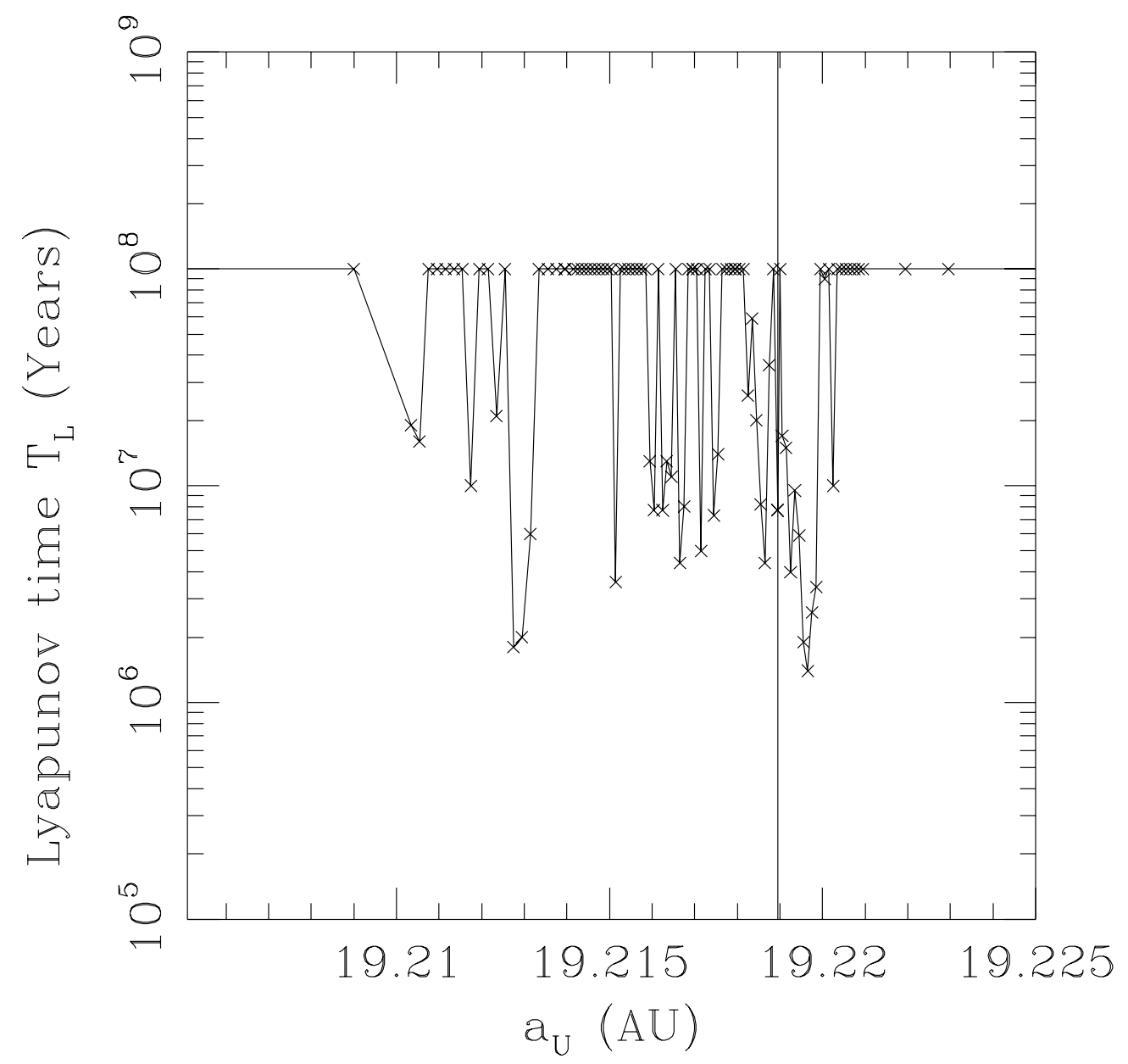




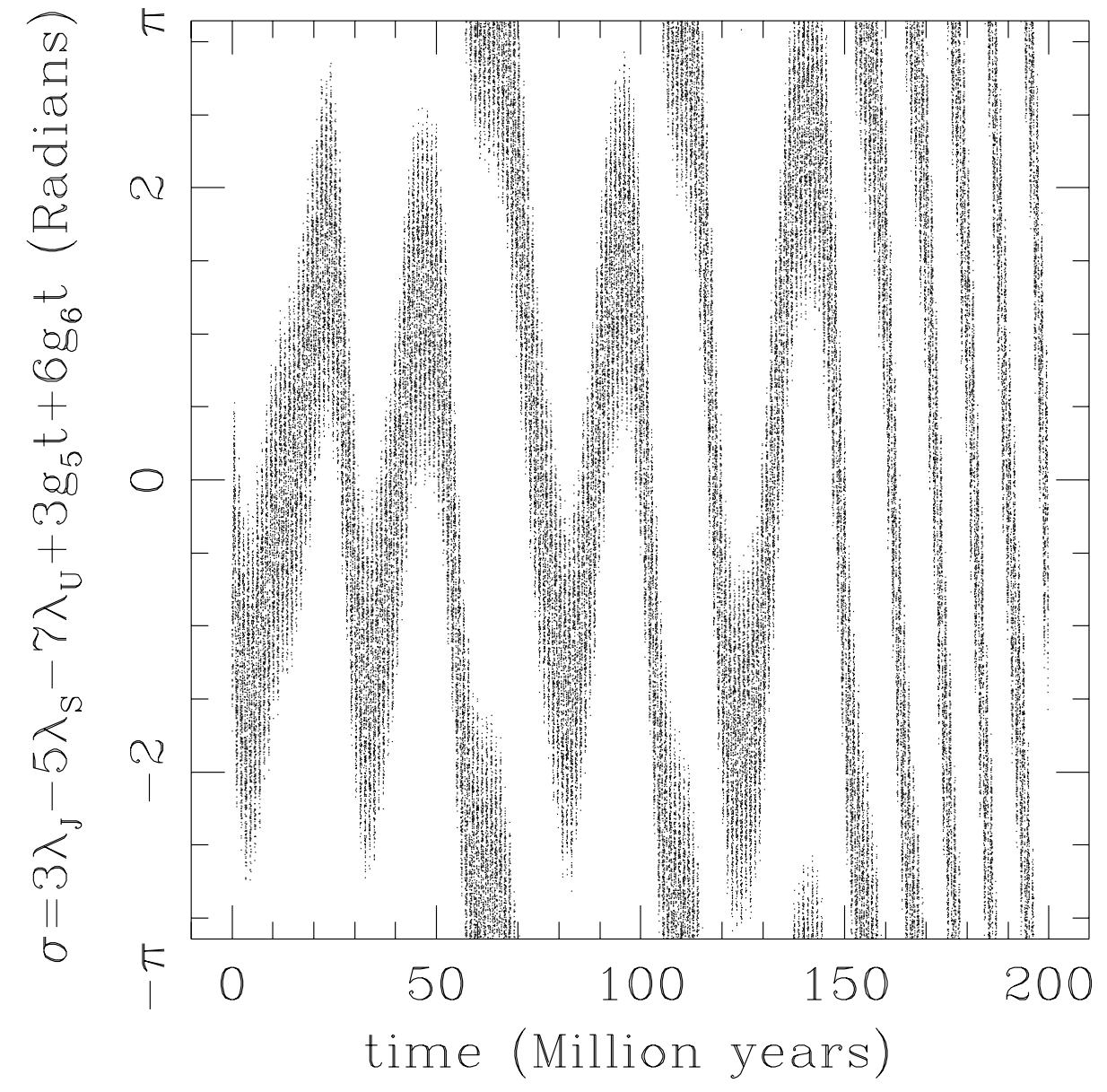

